\title{
A VISUALIZATION TOOL BASED ON TRAFFIC SIMULATION FOR THE ANALYSIS AND EVALUATION OF SMART CITY POLICIES, INNOVATIVE VEHICLES AND MOBILITY CONCEPTS
}

\author{
Lídia Montero \\ M. Paz Linares \\ Department of Statistics and Operations Research \\ and inLab FIB \\ Universitat Politècnica de Catalunya-UPC \\ Carrer Jordi Girona 1-3 \\ 08034-Barcelona, SPAIN
}

Oriol Serch

inLab FIB

Universitat Politècnica de Catalunya-UPC

Carrer Jordi Girona 1-3

08034-Barcelona, SPAIN

\author{
Josep Casanovas-Garcia \\ Barcelona Supercomputing Center, \\ Department of Statistics and Operations Research and inLab FIB \\ Universitat Politècnica de Catalunya-UPC \\ Carrer Jordi Girona 1-3 \\ 08034-Barcelona, SPAIN \\ Josep Casanovas-Garcia
}

\begin{abstract}
The CitScale tool is a software platform for visualizing, analyzing and comparing the impacts of smart city policies based on innovative mobility concepts in urban areas. It places emphasis on new automotive vehicles aimed at reducing traffic or environmental impacts. This paper introduces this traffic simulationbased tool, and two case studies developed for different scenarios in Barcelona City are briefly presented to demonstrate the capabilities of the tool when it is combined with microscopic traffic simulation software. The first case presents an extensive evaluation of new innovative vehicles (electric vehicles, bikes and three-wheeled scooters) and mobility concepts (trip-sharing). In the second one, data provided by connected cars is analyzed in order to compare different developed navigation strategies and how they affect the city. Finally, some of the obtained results from both cases are concisely presented in order to show the potential of the proposed tool.
\end{abstract}

\section{INTRODUCTION}

The CitScale tool is a software platform developed by inLab FIB at UPC for visualizing, analyzing and comparing experimental designs that model urban traffic modeling scenarios. Assessing new mobility concepts and new automotive vehicles are the main targets of projects that use CitScale. The core components of the CitScale analytics platform are a graphical interface and a traffic simulation model.

Traffic and transportation modeling follows the generic methodology involved in any model-building process (Barcelo 2010). Mobility is generated as a consequence of human activities distributed across space and time, thus person and freight movements arise. Traffic models are composed of demand and supply modeling. CitScale supports an aggregated representation of the demand based on origin-destination matrices between transportation analysis zones according to a time period and user class. User class refers 


\section{Montero, Linares, Casanovas-Garcia, Serch}

generically to either a purpose or a vehicle type or a combination of both. The supply is represented by a transportation network and its conditions of use in terms of traffic management and control policies. CitScale supports either microsimulation network models (very detailed representation), or mesocopic network models consisting of a representation of network geometry by means of intersections, links and turning movements. An explicit description of the traffic control policies for traffic lights and priority flows at intersections must also be considered. Additional objects such as detectors or variable message panels have to be allocated for realistic modeling objectives.

Transportation modeling platforms provide a wide range of graphical outputs and interfaces that run from basic to sophisticated. These are used to analyze results, but they are limited in terms of customization for comparison and joint description of basic Key Performance Indicators (KPI) for a set of replications related to an experimental design. CitScale is an interactive interface and it performs user-defined analytics on traffic simulation results after the completion of the executions, firstly from any browser and secondly without dependence on an active simulator license. CitScale simplifies the process of simultaneously accessing data results from different experiments and traffic scenarios. The system assists the analysts in evaluating and comparing developed scenarios from a basic model with the aim of understanding the effect of new mobility vehicle concepts and policies. To simplify the interpretation of scenario results, CitScale is based mainly on the development of geo-located map visualizations, which helps the analysts who work on this type of projects on a daily basis, but it also supports the demonstration for clients. A prototype for the assessment of static and time-dependent shortest paths has also been added and integrated.

The aim of this paper is to present a CitScale visualization and analytics platform through two projects developed at UPC, focusing on simulation-based concepts. The platform can be easily adapted to any traffic simulator with the required extended applications. AIMSUN (Transport Simulation Systems 2014) models were already available at UPC for the Barcelona Business District (L'Eixample) (Linares et al. 2016) and for the Barcelona Inner Urban Ring Road (Linares et al. 2017), both of which were integrated for supporting the experimental design of these two projects. The conceptual framework is described first. The next sections provide a description of both use cases while remarking on the analysis of the results obtained via the CitScale tool. Finally, the paper ends with conclusions and follow-up lines of research.

\section{SYSTEM FRAMEWORK AND ARCHITECTURE}

Figure 1 shows the proposed simulation testbed framework that is composed of an Executions Controller, a Traffic Simulation Module, a Result Preparation Module and a Visual Analytics Module. The visualization and analysis tool has been implemented using the Shiny (RStudio Project, n.d.) web application for $\mathrm{R}$ that simplifies the development of interactive web applications. The Shiny web application is agnostic to the traffic simulation platform. If we are going to emulate smart city policies by necessarily evaluating new vehicle types, probe vehicle sensors, navigation strategies and innovative mobility concepts such as multiple passenger ridesharing, then several ad hoc components in the traffic simulation model must be programmed using API extensions. However, any microsimulation platform that allows API extensions can be used.

The analysis of simulation results is performed by two fundamental and independent components: the Result Processing Module and the Visual Analytics Module. One deals with the automated preparation of data and the other with operating the data display.

The Result Processing Module in Figure 1 covers the entire pre-processing of data automatically, thus allowing the Visual Analytics Module to automatically use the simulation results directly without the need for any manual update. 
Montero, Linares, Casanovas-Garcia, Serch

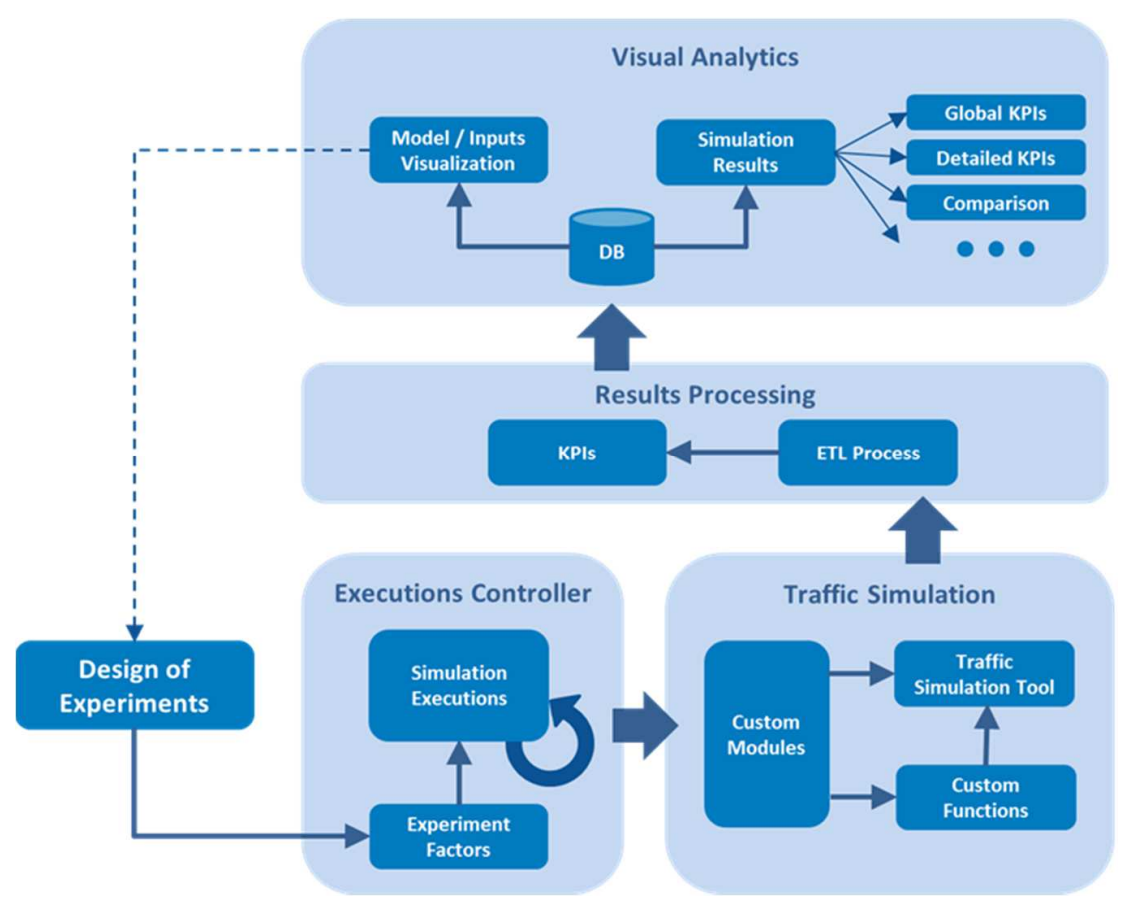

Figure 1. Simulation Testbed Architecture.

\subsection{Traffic Simulation Environment}

The traffic simulation environment of the framework (Figure 2) is composed of a traffic simulation tool, a set of auxiliary modules and functions that customize the smart mobility concepts to be evaluated, which is done by allowing the standard functionalities of the simulation tool to be extended in order to properly model the concepts to be evaluated. The simulation tool that is to be embedded into the framework must have certain capabilities such as:

- Representing the road network geometry properly.

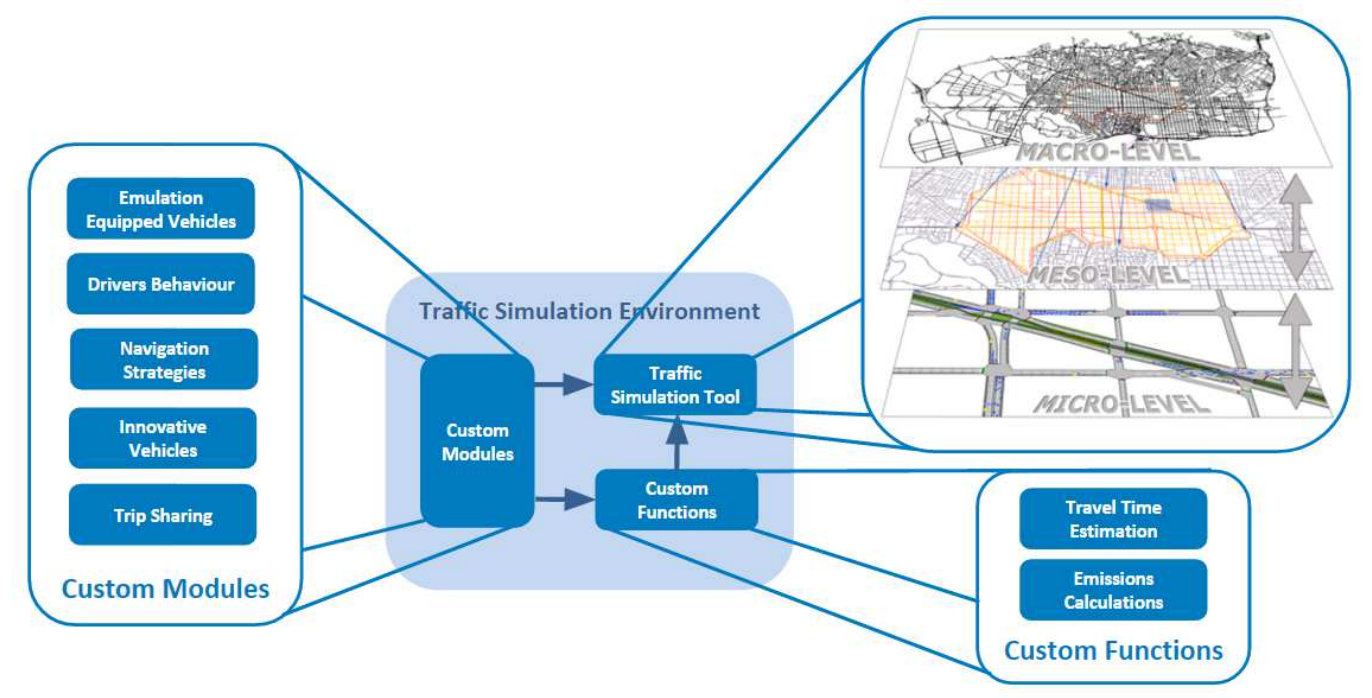

Figure 2. Traffic Simulation Environment. 
- Emulating the dynamic propagation of traffic flows on the road network. Vehicles in the simulation model travel from origins to destinations along the corresponding paths while taking into account space-time modeling.

- Dealing with several vehicle classes and allowing differentiated behaviors for them in order to account for the specific characteristic of the drivers and fleets of vehicles that are involved.

- Providing real-time functions to access and modify vehicle attributes and driver behavior during the simulation. These libraries should offer the possibility of obtaining information related to vehicles, nodes and the arcs on the graph representing the network topology, traffic lights, computed paths, etc. Furthermore, it is also of interest that the simulator can interact with external applications, either by using these very same interfaces or by any other method.

Different network representations and simulation approaches have been used: mesoscopic and microscopic. One of the two will be selected, depending on the necessities and expectations of the project:

- Innovative vehicles or mobility concepts that have been evaluated using a microscopic simulation framework are: electric bikes and vehicles, three-wheeled scooters, connected vehicles, different driver behavior, navigation strategies, fuel consumption, $\mathrm{CO} 2$ and $\mathrm{NOx}$ emissions, and multiple passenger ride-sharing based on the responsive transit concept of door-to-door demand.

- Mobility concepts that have been evaluated using a mesoscopic simulation framework are: tripsharing, OD Departure Adjustment (redistribution of the demand due to shifts in starting time of the trip in order to avoid high congestion periods) and park \& ride \& service areas to prevent city center access by car.

The exchange of information between the external application and the simulator is made at every simulation step, which is the time interval at which the state of the simulation model is updated. The programming languages in which Aimsun (Transport Simulation Systems 2014) provides its API are C++ and Python. While Python is used to easily collect some of the data, $\mathrm{C}++$ is needed for performance reasons to emulate the different applications.

The standard statistical results that are available after executing a simulation replication in SQlite DB format are also integrated into the CitScale platform.

\subsection{Executions Controller}

An experimental simulation consists of certain predefined numbers of replication executions. These replications are launched from a controller and a pre-process sets each of them up. At the end of the simulation, a post-process is run in order to collect the data generated during the simulation replications.

- Controller: This is responsible for launching the simulator. In addition, it manages the directory tree that stores the execution results and saves a history of all the executed simulations.

- Pre-run process: This computes previous calculations of the items, such as demand matrices, vehicle type configuration and others that depend on the specific experimental requirements.

- Post-run process: During this process, the selected KPIs are extracted from the output databases that are generated by the simulator. Additionally, other data required for the visual analytics features are also collected (sections and turning information, etc.)

\subsection{Results Processing}

The Results Processing Module (Salmerón-Moya 2016) in Figure 1 covers the automatic collection and pre-processing of the data before its visualization. This module is composed of an Extract- Transform-Load (ETL) process that is responsible for finding new execution results from recently performed simulation 


\section{Montero, Linares, Casanovas-Garcia, Serch}

replications and applying to them the processing that the Visual Analytics module needs. Specifically, the ETL process refers to extracting, transforming and loading the results of the traffic simulation executions. It performs the following actions: loading new simulation replication results, storing time-dependent and global KPIs provided by default by the simulator, calculating and storing KPIs not provided by default.

The output of the ETL process is a set of RDS files ( $\mathrm{R}$ internal single object files) in an $\mathrm{R}$ ( $\mathrm{R}$ Development Core Team 2016) dataframe-like format. This allows enhancing the speed of subsequent access, an improvement which is realized due to the lack of any need for directly accessing and processing data from the SQLites or CSV files generated by the simulation executions each time the Visual Analytics Module requires them. In fact, this preprocess reduces the time by $99.5 \%$ in comparison with performing direct access (Salmerón-Moya 2016).

\subsection{Visualization and Analytics}

The Visual Analytics Module in Figure 1 is based on (Lorente-García 2017) and covers the display of the simulation results from any previously replication execution. In addition, it allows the visualization of input data and other simulation model details. The visualization application is implemented with $\mathrm{R}$ in the framework of Shiny (RStudio Project, n.d.), which allows development without knowledge of web code languages. C++ applications, HTML, CSS and JS have been developed for enhancing optimal performance.

Currently, the Visual Analytics Module offers the possibility of analyzing and comparing on a timedependent basis the following Key Performance Indicators (KPIs): total travelled distance (km), total travelled time (h), total liters of fuel consumed, $\mathrm{CO} 2(\mathrm{~kg}), \mathrm{NOx}(\mathrm{kg})$, average travel time $(\mathrm{sec} / \mathrm{km})$, density $(\mathrm{veh} / \mathrm{km})$, average speed $(\mathrm{km} / \mathrm{h})$, average flow $(\mathrm{veh} / \mathrm{h})$, throughput rate $(\%)$ completed trips divided into total number of trips for $1 \mathrm{~h}$. The addition of new KPIs has been considered in the design of the module.

This module provides several possibilities for analyzing traffic simulation results aimed at assessing smart mobility concepts and vehicles. A brief description of some of them can be found as follows.

1. Global KPI analysis: This component shows different summary tables for all the simulation scenario results for an experimental design (Figure 3). KPIs are obtained by combining results obtained for all replications of each configuration design. A first table displays the global values for each defined KPI and scenario, and two additional tables display relative and absolute differences between each configuration with respect to the base scenario configuration.

2. City network impact: This analysis allows the visualization of the KPI results of a simulation replica over the current network model. It shows the selected KPI values at link level for each simulation interval and the overall simulation horizon.

3. Analysis of the trips: This analysis allows the visualization of all the paths used by drivers/vehicle classes in the simulations on a time-dependent basis. A line-path visualization renders all the streets included in origin-destination paths for each drive/vehicle class (Figure 4).

4. Mobility patterns: This feature shows a visualization and analysis of all horizon mobility patterns (origins and destinations of the trips). The trips among origin-destination pairs are displayed, as well as the demand generation and attraction of each centroid (Figure 5). This display is shown at the vehicle class level and overall. The most used paths are clearly highlighted.

\section{CASE STUDY 1: EVALUATION OF NEW VEHICLES AND AN INNOVATIVE MOBILITY CONCEPT}

The objective of the first presented case study is to evaluate the pervasive penetration of new vehicles and mobility concepts by measuring the effect of the:

- Replacement of fossil fuel propelled vehicles by vehicles with more efficient engines propelled by alternative or green energy sources. 


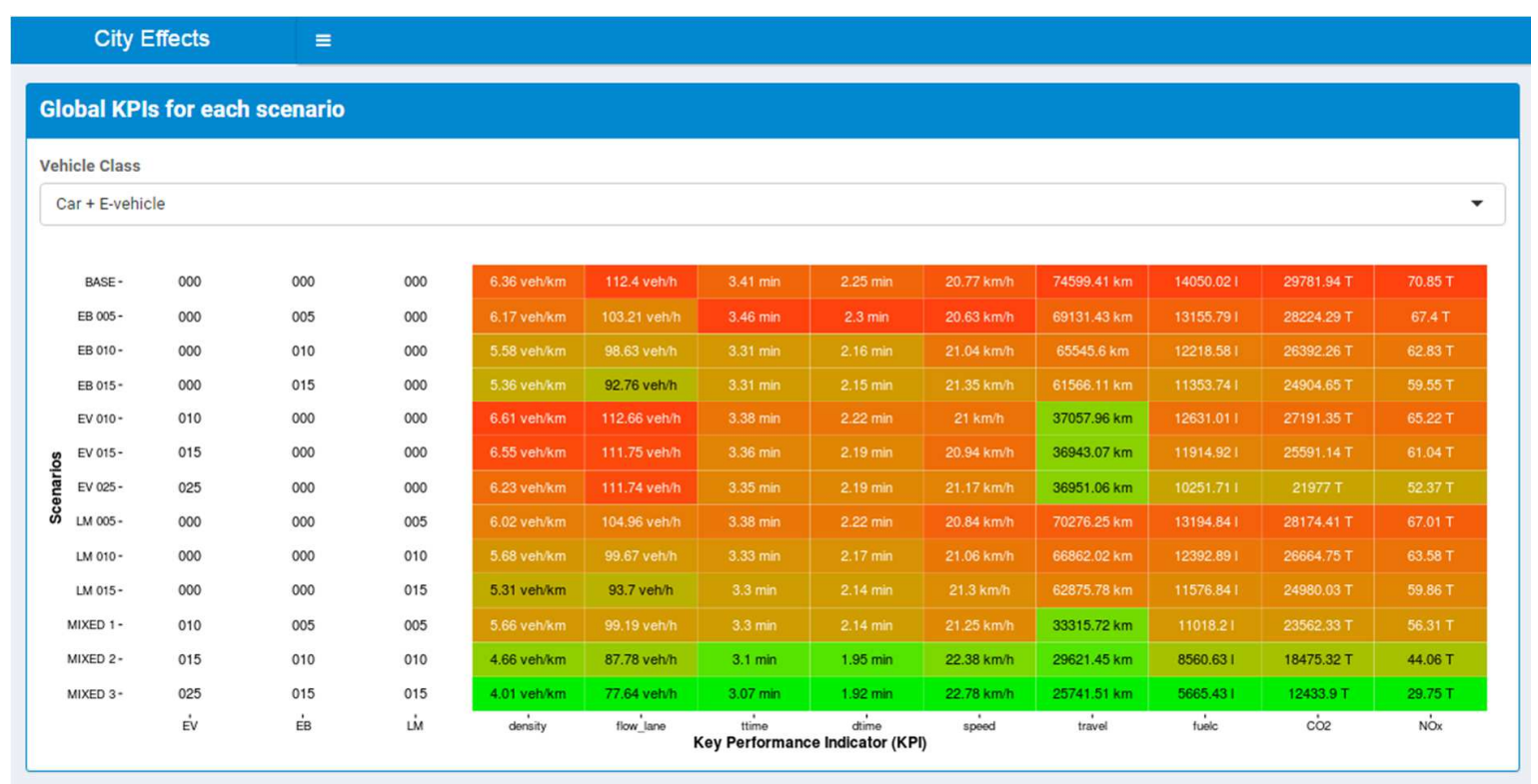

Figure 3. Comparison to Base Scenario for each Vehicle Concepts Configurations.

- Urban policies committed to helping citizens use bicycles and to making them safer, as a key component of sustainability action plans. The shift from private car to shared bicycle systems and lightweight three-wheeled electric scooters, usually involves establishing a segregated bike network.

- New mobility concepts such as those under the Mobility-as-a-Service paradigm, car-sharing, or multiple passenger trip-sharing. These describe a shift from personally owned modes and towards mobility solutions as a service. Transport information together with telecommunications infrastructures allow development of real-time systems to turn mobility into a service.

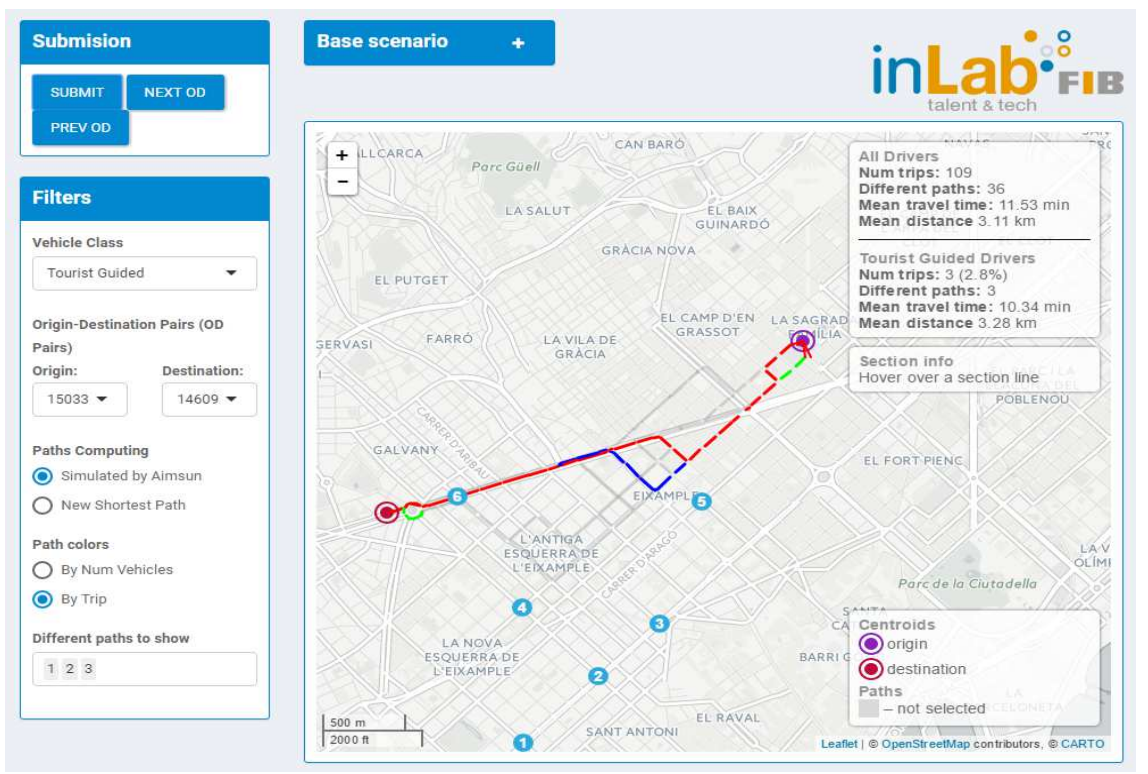

Figure 4. OD paths for a selected OD pair in the Base Scenario. 


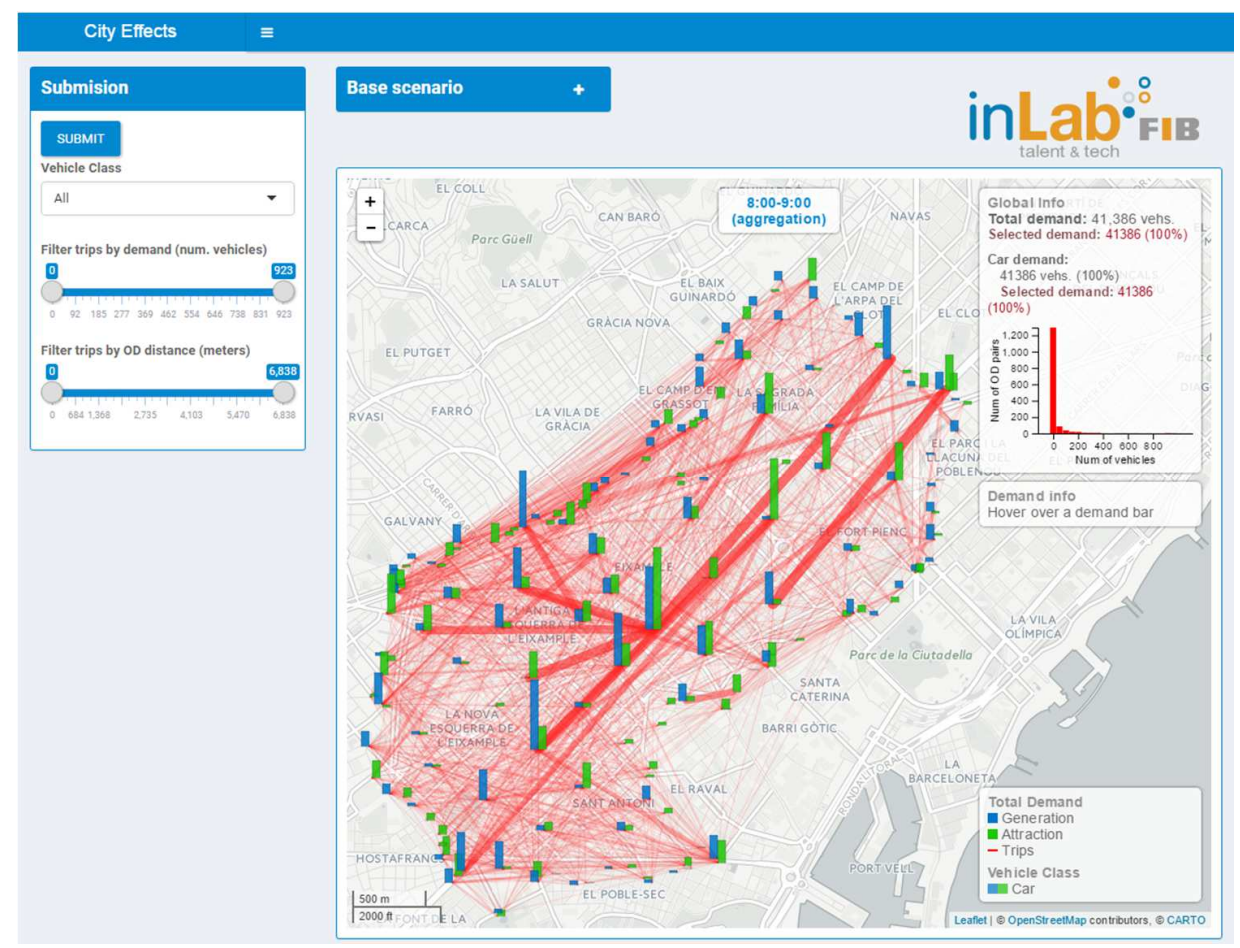

Figure 5 : OD flows, total generated/attracted trips in Base Scenario for a subset of OD pairs.

\subsection{Custom Modules}

In order to extend the standard functionalities of the selected simulation tool so that it can deal with the specific requirements of this case study, vehicles and innovative mobility concepts have been implemented into the Custom Module (Figure 2).

\subsubsection{Design of Experiments for Microsimulation modeling: Innovative Vehicles and Concepts}

The innovative vehicles and concepts that have been evaluated using a microscopic simulation framework are:

- Electric vehicles: The car fleet profile according to emissions for the modeled urban area is modified by including a new electric car fleet with no emissions. A 50-50 petrol-diesel EURO3 Emission Standard fleet for non-electric vehicles was assumed in the base scenario. Emissions and fuel consumptions are modeled according to (Panis, Broekx, and Liu 2006). Core model parameters (car-following, lane-changing and gap-acceptance parameters) in traffic microsimulation for this vehicle class have been carefully specified. For example, vehicle type parameters have been defined as a truncated normally distributed length with mean $4 \mathrm{~m}$ ( 0.5 standard deviation) and $\mathrm{min} / \mathrm{max}$ thresholds $3.5 \mathrm{~m} / 4.5 \mathrm{~m}$, vehicle width as $1.70 \mathrm{~m}$; car-following and lane-changing parameters as:

○ Desired speed between 40 and $70 \mathrm{~km} / \mathrm{h}$, truncated normally distributed around $60.0 \mathrm{~km} / \mathrm{h}$ (standard deviation $10 \mathrm{~km} / \mathrm{h}$ )

- Speed acceptance (between 0.9 to 1.30 , normally distributed at around 1.1) This parameter $(>0)$ can be interpreted as the degree of acceptance of speed limits.

○ Look-ahead distance for lane selection: around $250 \mathrm{~m}$ in urban streets, $500 \mathrm{~m}$ in arterials and motorways. Minor streets $100 \mathrm{~m}$. 
- Inter-vehicular distance: truncated normally distributed around $1 \mathrm{~m}$ with standard deviation of $0.3 \mathrm{~m}$. Min/Max $0.5 \mathrm{~m} / 1.5 \mathrm{~m}$

Design levels for globally shared electric vehicles are established at 10\%, 15\% and $25 \%$.

- Electric bikes have an OD pattern demand obtained as a percentage of the global OD pattern when Origin and Destination access to bike lines is less than $500 \mathrm{~m}$. Design levels for capturing those trips are selected at $5 \%, 10 \%$ and $15 \%$.

- Three-wheeled scooters have an OD pattern demand obtained as a percentage of the global OD pattern when Origin and Destination accesses to bike lines is less than $500 \mathrm{~m}$ and the total trip length is less than $1.5 \mathrm{~km}$. Design levels for capturing those trips are selected at $5 \%, 10 \%$ and $15 \%$.

- Multiple Passenger Ridesharing mobility concept penetration are 5\%, 10\% and $15 \%$. The morning period OD pattern was an additional design factor.

\subsubsection{Design of Experiments for Mesoscopic simulation modeling: Trip Sharing}

Design factors for the Metropolitan scenario considered electric vehicle penetration and several innovative mobility concepts such as Trip Sharing, OD Time-Adjustment, etc. Since a detailed description of all factors is beyond the scope of this paper (due to length), just one of the concepts involved in the experimental design is detailed: the Trip Sharing Factor. The aim of trip sharing is to reduce the number of cars in the network and thus producing less emissions and congestion. The emulation of a car sharing service based on sharing one or more car trips and by showing proximity on OD commuting trips has been evaluated using a mesoscopic simulation framework. The trip sharing mesoscopic modeling is affected by three parameters:

- Alpha_acceptance: percentage of the total number of trips suitable for Trip Sharing at the OD pair level (tested levels $0 \%, 15 \%$ and $30 \%$ ).

- Beta_occupancy: maximum number of passengers for shared vehicles (to be specified, pax/car).

- Interval length $\mathrm{T}$ : to be specified for grouping common trips with respect to departure time and OD.

As a consequence, total OD car trips were reduced to 666,029.72 (15\%) and 581,603.40 (30\%) for a max passenger per car set at 4 pax/car and interval length for grouping purposes at $15 \mathrm{~min}$.

\subsection{Analysis of the Simulation Experiments using the Visual Analytics Tool}

Project realization and visual analytics development were based on the "L'Eixample" area (which comprises $7.46 \mathrm{~km}^{2}$ and more than 250,000 inhabitants) and the Inner Ring of the Barcelona Metropolitan Area (see Figure 6) (which comprises $102 \mathrm{~km}^{2}$ and more than 1,700,000 inhabitants). The model consists of 1,720 links, 528 nodes, 120/130 generation and attraction centroids, and 877 non-zero OD pairs with a horizon study of $1 \mathrm{~h}$, accounting for a total number of 41,400 vehicle trips. And the model of the Inner Ring Area consists of 16,000 links, 8,000 nodes, a horizon of $4 \mathrm{~h}$, accounting for a total number of 350,000 trips.

The microscopic traffic simulator updates the model state every 0.5 second, specifically the positions of all vehicle classes considered: passenger cars, buses and ride-sharing vehicles. Passenger car demand is modeled as 15-min time-sliced demand whose OD pattern reproduces one hour of a working day morning period in the Eixample. A 4h morning period is selected for mesoscopic simulation in the Metropolitan model, and the global KPI displayed with the CitScale platform is presented in Figure 7. 


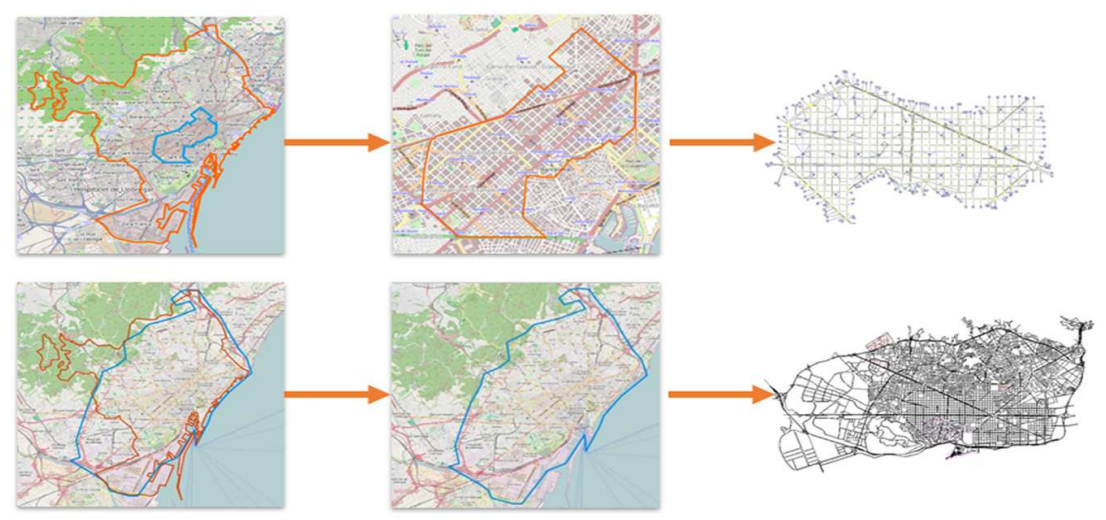

Figure 6. Top: Eixample area (left), simulation test-site (center), and the Aimsun model (right). Bottom: Inner ring area of Metropolitan Barcelona (left), simulation test-site (center), Aimsun model (right).

\section{CASE STUDY 2: PROBE VEHICLE DATA AND NAVIGATION STRATEGIES}

In urban areas, equipped vehicles with onboard sensors are expected to reach high concentrations in the near future. This offers the possibility of enabling a new family of services based on these advances in intervehicular communications. In particular, the proposed case study aims at evaluating the effect of using probe car fleet data at different penetration levels to develop real-time navigation strategies for connected vehicles. This requires detailed driver behavior, heuristic route choice modeling that depends on knowledge of the network and congestion, and the availability of navigation data. Traffic microsimulation capabilities are needed to achieve the final goal.

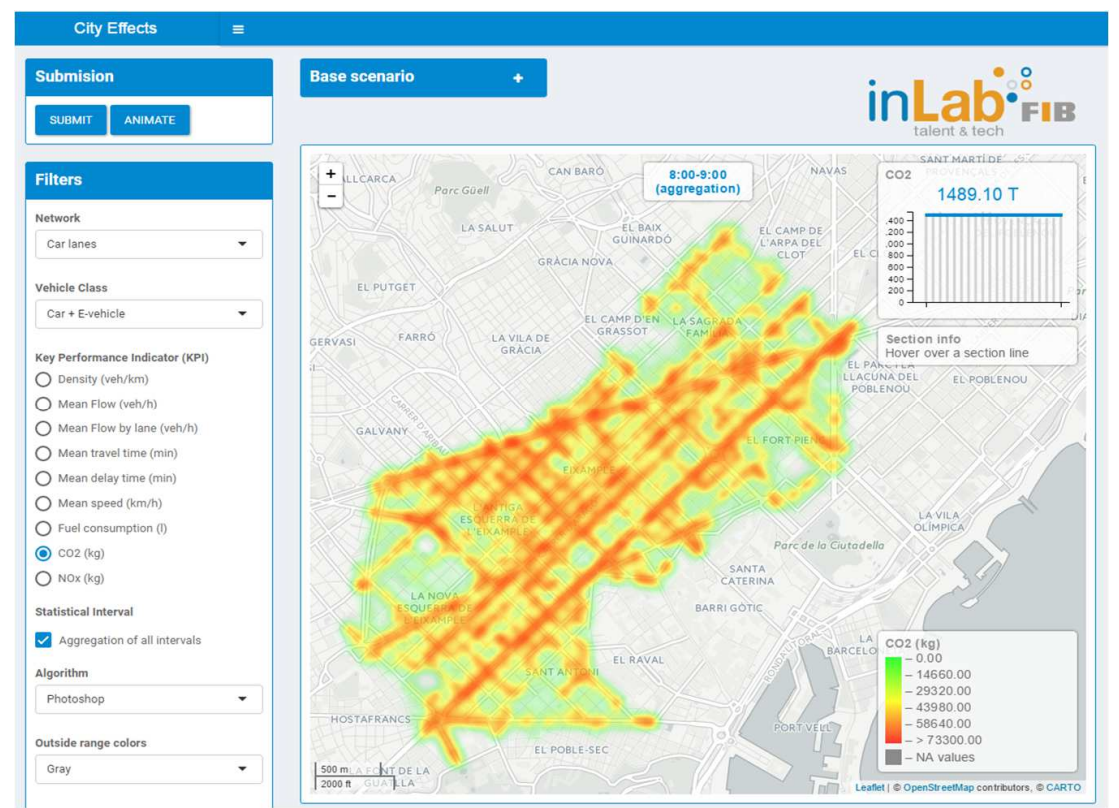

Figure 7. Metropolitan Area: Global Emissions KPI.

Comparison to Base Scenario Concept design and KPI display. 


\subsection{Custom Modules}

To extend the standard functionalities of the selected microsimulation tool so that it can deal with the requirements of this case study, the next items have been implemented into the Custom Module.

- Probe car data: the emulation of cars with equipped sensors (V2I and V2V technology) connected to a Traffic Management Center. This work assumes that the V2X and V2V technology is on board probe cars (Montero et al. 2016). Collected data were analyzed and cleaned to remove any outliers and incomplete observations. The filtered data were then used to calibrate the emulation of PVD by an API included in the Traffic Simulation Module. Only basic vehicle sensors were considered in the current case study (position and speed).

- Driver's behavior: the emulation of different profiles of drivers according to network congestion knowledge and availability of navigation. Drivers are classified into three groups: tourists (low knowledge), regular drivers (good path and congestion knowledge for recurrent trips and poor performance for occasional trips) and expert drivers (excellent network and congestion knowledge for the day-to-day trips occurring in the morning period and recurrent trips). For each group, the availability of navigation guidance based on actual traffic conditions at different levels was a design factor in the simulation experiments.

- Navigation strategies: the emulation of heuristics for routing strategies by differentiating from among those based on either link or lane travel time estimates, which themselves are based on probe car data. Three strategies were tested according to navigation assistance that is based on free-flow travel times, link travel time estimates and lane travel time estimates. Estimates are input to the stochastic route choice model, which aims to characterize the origin-to-destination paths that are used and the proportions in the Eixample.

- Time-dependent link and lane travel time estimation module that uses data collected by PVD. A proposal based on (Sanaullah, Quddus, and Enoch 2013) has been implemented.

\subsection{Design of Experiments}

Microscopic modeling for the "L'Eixample" (see Section 3.2) area is considered. The simulation experimental design has considered the following factors:

- Driver type distribution into Expert-Regular-Tourist.

- Guidance penetration: Percentage of total cars that are connected cars whose route choice decisions follow those advised by a navigation system.

- Demand pattern: This refers to a perturbation of the historic demand pattern of OD pairs that belong to the fourth percentile trip distance.

- Probe Vehicle penetration percentage. This fleet collects real-time data through their sensors.

- Time-window length is the rolling horizon interval considered for the estimation of traffic variables from PVD.

- Navigation strategy models driving recommendations based on either lane-level or link-level connected car data.

\subsection{Analysis of the Simulation Results using the Proposed Visualization Tool}

Experimental results for the whole design have been integrated into the CitScale tool. A detailed analysis on the effects of factors used in the experimental design was undertaken using R, and some of the most attractive graphical plots are in the process to being incorporated into the CitScale tool. For example, the Spearman correlation coefficient for full-horizon simulation and all driver types is shown in Figure 8, where an intense correlation between KPIs is highlighted. 


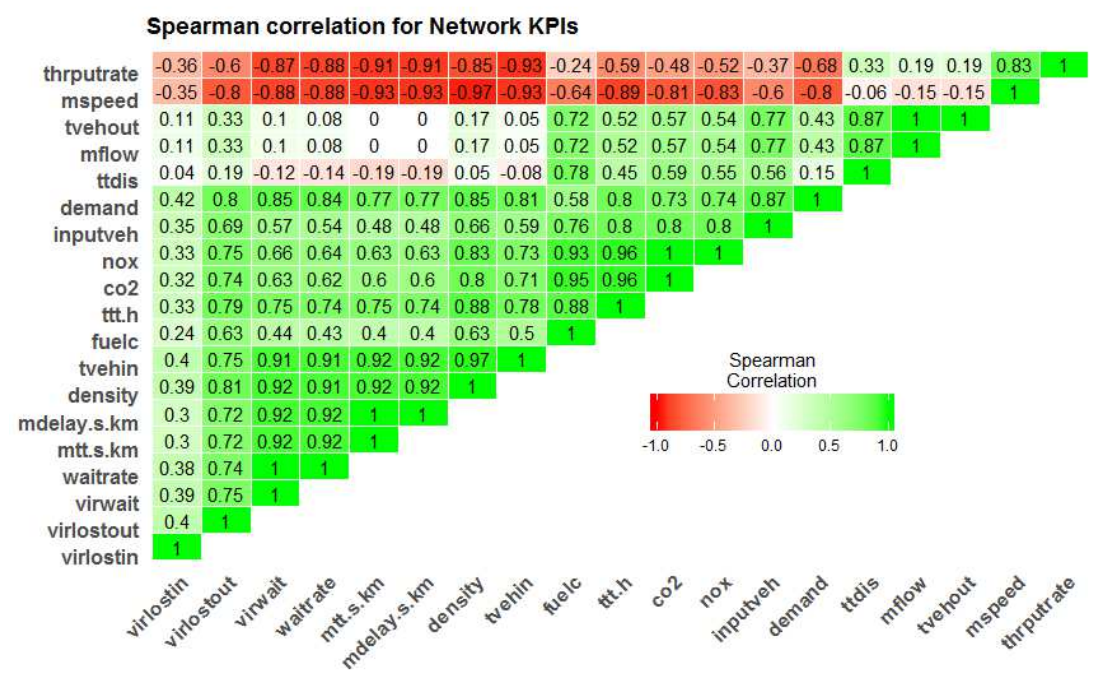

Figure 8. Spearman correlation for selected KPIs from simulation experiments.

The overall KPIs are affected mostly by Demand Pattern factors and Guidance Penetration. Although Navigation Strategy, Interval Length for Estimation and PVD factors are significant elements in the system performance (level of service), they are less important. A second design for gaining insight into the critical factors has been launched.

\section{CONCLUSIONS AND FUTURE RESEARCH}

The CitScale visual analytics platform has proven to be very practical in supporting the analysis of results for different experimental designs. Designs based on different traffic simulation models and different purposes have been incorporated. The first presented project roughly focuses on analyzing the impact of emissions-related factors on KPIs in the Eixample, where a shift to more green modes such as bicycles has been shown in Case Study 1. A project for the evaluation of the Probe Vehicle Data use has been incorporated into CitScale (Case Study 2), and some detailed results have been shown. This flexible tool will save a lot of time for analysts who work on traffic and transportation projects, and it will be further developed in the future at UPC. A further development for improving the demand modeling in CitScale will rely on a supporting representation of Activity Based Demand. The possibilities and flexibility of the Visual Analytics Tool allow for a diverse set of projects, thus the list of future developments for enhancing the analysis capabilities is extremely long.

\section{ACKNOWLEDGMENTS}

This research was funded by TRA2016-76914-C3-1-P of the Spanish R+D National Programs and by the Secretaria d'Universitats i Recerca de la Generalitat de Catalunya under 2014 SGR 1534. Throughout, the authors have benefited from the support of R.M. Martín, E. Lorente, G. Navarro, G. Recio and J. Salmerón from inLab FIB (UPC).

\section{REFERENCES}

Barcelo, Jaume. 2010. Fundamentals of Traffic Simulation. Simulation. Vol. 145. doi:10.1007/978-1-44196142-6.

Linares, M. P., L. Montero, E. Lorente, O. Serch, G. Navarro, J. Salmerón, and J. Casanovas-Garcia. 2017. "Analytics Tool for Assessing Innovative Mobility Concepts, Vehicles and City Policies (CitScale)." 
In Proceedings of the 5th IEEE International Conference on MODELS AND TECHNOLOGIES FOR INTELLIGENT TRANSPORTATION SYSTEMS, 1-6.

Linares, M.P., L. Montero, J. Barceló, and C. Carmona. 2016. "A Simulation Framework For Real-Time Assessment Of Dynamic Ride Sharing Demand Responsive Transportation Models." In Proceedings of the 2016 Winter Simulation Conference T. M. K. Roeder, P. I. Frazier, R. Szechtman, E. Zhou, T. Huschka, and S. E. Chick, Eds.

Lorente-García, E. 2017. "Sistema de Visualización de Datos Procedentes de Resultados de Experimentos de Simulación de Tráfico." Universitat Politècnica de Catalunya. http://upcommons.upc.edu/handle/2117/103220.

Montero, Lidia, Meritxell Pacheco, Jaume Barceló, Silviu Homoceanu, and Josep Casanovas. 2016. "Case Study on Cooperative Car Data for Estimating Traffic States in an Urban Network." Transportation Research Record: Journal of the Transportation Research Board 2594 (January). Transportation Research Board: 127-37. doi:10.3141/2594-16.

Panis, Luc Int, Steven Broekx, and Ronghui Liu. 2006. "Modeling Instantaneous Traffic Emission and the Influence of Traffic Speed Limits." Science of The Total Environment 371 (1): 270-85. doi:10.1016/j.scitotenv.2006.08.017.

R Development Core Team. 2016. "R: A Language and Environment for Statistical Computing." $R$ Foundation for Statistical Computing. doi:10.1007/978-3-540-74686-7.

RStudio Project, R. n.d. "Shiny Web Application Framework for R." https://www.rstudio.com/products/shiny/.

Salmerón-Moya, J. 2016. "Design a BigData Architecture for Simulation Environments (in Spanish)." Universitat Politècnica de Catalunya (UPC). http://hdl.handle.net/2117/91397.

Sanaullah, I., M. Quddus, and M. Enoch. 2013. "Estimating Link Travel Time from Low-Frequency GPS Data." In Proceeding of the 92nd Transportation Research Board Annual Conference. Proceeding of the 92nd Transportation Research Board Annual Conference Washington D.C.: Transportation Research Board. https://trid.trb.org/view.aspx?id=1242269.

Transport Simulation Systems. 2014. “Aimsun Microscopic Simulator (v 8.0).” http://www.aimsun.com.

\section{AUTHOR BIOGRAPHIES}

LIDIA MONTERO obtained her PhD in Computer Science in 1993 at the Universitat Politècnica de Catalunya (UPC). She is now Associate Professor at the Department of Statistics and Operations Research at the UPC. Her research concerns simulation-optimization issues, demand modeling, data analysis and models with applications in transport. Email: lidia.montero@upc.edu. Web Page: http://futur.upc.edu/LidiaMonteroMercade.

$\mathbf{M}^{\text {a }}$ PAZ LINARES is a postdoc researcher at the inLab FIB (Barcelona informatics school laboratory) in the area of Mathematical Programming, Logistics and Simulation. Her research concerns dynamic traffic assignment, traffic simulation and optimization. Email: mari.paz.linares@upc.edu.

JOSEP CASANOVAS-GARCIA is a full professor in Operations Research, specializing in Simulation systems. He is one of the founders of the Barcelona School of Informatics.He is also the director of inLab FIB.. His email address is josepk @ fib.upc.edu.

ORIOL SERCH is a researcher at inLab FIB UPC in the Smart Mobility group, collaborating in projects related to dynamic traffic models and data analytics. He obtained his Degree in Computer Science from the UPC in 2008 and completed the Master's degree in Statistics and Operations Research in 2014. His email address is oriol.serch@upc.edu. 